\title{
Vida, afectividad y racionalidad. Hegel y la biología filosófica de Scheler
}

\author{
JOSÉ IGNACIO MURILLO \\ Universidad de Navarra
}

En su Curso de Psicología general, dictado en los años setenta y recientemente publicado, Leonardo Polo incluye a Hegel como uno de los autores clave en el alumbramiento y en los avatares de "lo psíquico" (L. Polo 2009, pp. 55-168). "Lo psíquico" es, según Polo, "un punto de vista dinámico respecto de temas". Con esta noción aparece una nueva forma de entender el hombre y sus dinamismos. No se trata ya del alma socrática, ni de sus versiones aristotélica y neoplatónica. La tradición socrática interpretó el alma, su actividad y su lugar en la realidad desde los descubrimientos precedentes del logos o del nous (cfr. J. I. Murillo 2005, pp. 7-16). Para Sócrates, el alma se identifica con el yo, entendido ante todo como agente moral, que busca y hace el verdadero bien, y este sólo es el que se presenta como tal ante su intelecto. El alma es el principio de unidad del hombre y es capaz de ordenar el deseo humano desde y hacia el conocimiento del verdadero bien, que es la más alta forma de posesión. El deseo, la hórexis, no es nada al margen del conocimiento. Toda aspiración se ordena en último extremo hacia la contemplación. De ahí, por ejemplo, que Aristóteles identifique lo más divino con el acto de entender y que lo más cercano que haya en Plotino a una caracterización positiva del Uno sea hablar de él como "cierta contemplación” (cfr. G. Reale 1989, pp. 612-616).

La total subordinación del deseo al noûs y a la contemplación (theoría) comienza a matizarse en las filosofías que reciben con más fuerza el influjo del cristianismo. En ellas la voluntad se destaca como una instancia que no puede ser totalmente reducida a la contemplación intelectual, y que es origen de una actividad propia y peculiar. De hecho, esta noción, que al parecer no se formula hasta el estoicismo tardío (cfr. M. Pohlenz 1992, pp. 319-320), será explotada sobre todo por el pensamiento cristiano, que subraya la originalidad (en el sentido de originariedad) del yo en el acto moral. Una originalidad que no responde, como parece sugerir Aristóteles, a un defecto de ordenación por parte de éste, sino a algo positivo y activo, capaz a su vez de introducir orden.

Pero hay que esperar hasta la Baja Edad Media para que el dinamismo 
humano se emancipe de la razón o inteligencia. Para Duns Escoto, la voluntad es espontánea y no debe esperar para determinarse a la razón, sino que se determina por sí misma a obrar. La voluntad, se podría decir, parafraseando a Pascal, tiene "razones" que la razón no conoce. ¿Hasta qué punto es legítimo designarlas con este nombre? Éste es precisamente uno de los aspectos de la historia que nos ocupa.

La idea de que hay un dinamismo anterior a la razón y la objetividad abre el camino, según Polo, a la noción de "lo psíquico". Es claro que aparece de este modo una instancia independiente respecto de las ideas, que puede interferir con la lógica, como se ve en el voluntarismo posterior. En Platón y en Aristóteles, la objetividad de las ideas está gobernada por reglas internas que no están sujetas al arbitrio de nadie. Su estudio es, para Aristóteles, el objeto de la lógica. En Descartes, la voluntad es capaz de controlar los objetos para someterlos a sus exigencias: en especial, a la búsqueda de la certeza. Pero tampoco aparece lo psíquico con precisión porque el individuo se identifica con la voluntad y es ésta la que somete activamente a la objetividad. Para Descartes, las ideas son presentadas como un límite para la voluntad, y ésta se define por su capacidad de producir.

El racionalismo posterior intentará recuperar la lógica a costa de la voluntad, disolviendo esta última en aquella. Esto se nota con especial intensidad en Spinoza. En él todo se resuelve en el "orden y conexión de la ideas". Fuera de ese orden no hay nada relevante. Ni siquiera la existencia. Descartes se vio obligado a añadirla al cogito como algo externo e indeducible; pero la deriva racionalista de su propuesta se manifiesta en que intenta recuperarla para el sistema acabado de la razón con su argumento a simultaneo para probar la existencia de Dios, en el que la existencia se encuentra contenida en la idea del ser infinito.

En el empirismo, en cambio, y en particular en Hume, las ideas son gobernadas por un dinamismo previo, que sólo puede ser considerado nuestro de un modo vago, es decir, en la medida en que precede a las ideas que se nos presentan. En Hume es ya claro que ese dinamismo previo no permite afirmar la realidad del sujeto o del yo.

La afirmación de que la conexión de las ideas depende de un dinamismo no racional desemboca en el escepticismo. Junto con él, el hombre aparece gobernado por una instancia no racional, que podemos denominar «afectividad». No es extraño que la moral no dependa en Hume de la razón, que, en su opinión, sólo sirve para hablar de los hechos y su conexión: la valoración es extrarracional.

En este estado de cosas, cabe interpretar la filosofía kantiana como un intento de eliminar la arbitrariedad que introduce esa instancia que precede a la objetividad y a las conexiones entre los objetos. Estudiar el proceso de formación 
de las ideas y hallar en él algo que no es arbitrario es un modo de recuperar la racionalidad. Una racionalidad que, a diferencia de la racionalista, ya no se presenta como meramente objetiva, sino como un proceso de construcción por parte de una subjetividad. La Crítica de la razón pura nos habla, en efecto, del modo como se construye el objeto, pero esto no ocurre mediante un proceso ajeno a la necesidad y universalidad de la razón. A la espalda de la actividad espontánea de la razón vertida sobre la experiencia sensible se encuentra una espontaneidad inobjetivable, pero que no es arbitraria, que construye siguiendo reglas precisas, un sujeto que no es empírico: el sujeto trascendental.

Este intento de recuperar la hegemonía de la razón - una hegemonía que, sin embargo, es precaria - , salta a la vista en su interpretación racionalista del deber, que consiste en ser interpelado por la razón e instado a elevarse a su altura. Lo que se intenta conjurar de este modo es el irracionalismo que está en la base de la moral de Hume. Es más, la afectividad queda en Kant totalmente marginada; podríamos decir, incluso, reprimida. Conceder un papel positivo a la afectividad sería como separarse de la razón y recaer en la arbitrariedad, resignarse a ser guiado por un dinamismo irracional, mientras que, para Kant, sólo a lo racional, y a la necesidad que lo acompaña, se encuentra vinculada la dignidad que corresponde al hombre y que éste, con su conducta, intenta realizar.

Hegel representa en esta historia el intento de ir más allá de Kant en esta lucha contra la arbitrariedad de "lo psíquico". Para él también hay una espontaneidad en el inicio y también, por encima de la lógica formal, positiva, existe una lógica que corresponde al proceso que alumbra los contenidos del saber y que desemboca en la Idea absoluta. Pero el fin del proceso no es, como en Kant, la constitución de la ciencia, sino la Idea, el Absoluto mismo.

Hegel es más radical que Kant también a la hora de tratar la lógica formal. Ésta, en tanto que mantiene separados los conceptos, no puede ser la lógica verdadera. Polo interpreta la naturaleza del proceso dialéctico y su pretensión de generar nuevos contenidos sentando, entre otras, las siguientes tesis:

1. El proceso no puede ser lo definitivo, sino que se encuentra entre la inmediatez, que es anterior al primer momento de la lógica, y la contemplación de la Idea, que es el cuarto momento.

2. El primer momento abre paso al segundo momento - la negación-, que es para Hegel el mecanismo para conseguir una pura novedad. Ahora bien, para que la negación aporte algo, el contenido que genera debe estar, a su vez, separado de lo que se niega. Esa separación es su «estar puesto», su positividad.

3. Es el hecho de que A y no A estén puestas, y, por lo tanto, separadas, lo que permite y exige el tercer momento, cuya misión es precisamente suprimir la separación entre ambas anulando la positividad, que, para- 
dójicamente, es lo que ambas tienen en común. De este modo se alcanza

la unión concreta de ambos contenidos, su mutua circulación.

Pero la posición o separación entre los contenidos, que se hace patente con la primera negación, es, para Hegel, lo falso. Esto quiere decir que la falsedad forma parte del proceso, pues, sin ella, no habría tercer momento ni, por lo tanto, proceso generativo alguno. El proceso dialéctico es un método que pretende generar nuevos contenidos y reunirlos anulando o despejando su separación. Pero esto sería imposible si la razón no se resignara a soportar la falsedad que inevitablemente introduce la primera negación. Ahora bien, si la falsedad forma parte del proceso racional, la racionalidad, para Hegel, no se opone tanto a la falsedad como a la irracionalidad, donde la irracionalidad es ante todo la positividad, la separación entre los contenidos.

Nos encontramos así ante una paradoja. La negación es un método que consigue un contenido nuevo, pero este no puede objetivarse o delimitarse al margen de aquello que niega. Además, si, en el segundo momento, no A no aparece separado de A, entonces A y no A se convierten en pura respectividad y su contenido se anula, es decir, no puede ser pensado. Recordemos que, para Hegel, el principio lógico formal de identidad no rige, es decir, no podemos decir que "A es A". Esta identidad falsa sólo puede establecerse en la medida en que A es dominado por la positividad. Pero, como hemos visto, la positividad es el principio de la separación y, por lo tanto, de la falsedad. Cabe decir que, en esta expresión, "es" equivale a falsedad y es lo que el proceso pretende eliminar. Por eso tiene sentido que aparezca no A. Ahora bien, si no A no se encontrara puesta del mismo modo en que A lo estaba, el nuevo contenido sólo se podría establecer respecto de $\mathrm{A}$, y hasta tal punto serían inseparables que la relación resultaría reversible y, de este modo, ininteligible. Sería un puro trance, pero nada propiamente pensable. Por otra parte, la eliminación prematura de la positividad anularía el proceso generador de contenidos. Lo haría imposible y, por lo tanto, eliminaría el camino hacia un saber absoluto, hacia una completa claridad en la que el sujeto comparezca totalmente, se reconozca.

Polo asimila la situación que derivaría de eliminar prematuramente la positividad y, con ella la separación entre contenidos, a una "afectividad sola". Hegel rechaza que la inmediatez sea la afectividad. Esto sería para él la alienación máxima: la pérdida del estatuto de objeto, la invasión de lo arracional. $\mathrm{Su}$ lucha contra la irracionalidad contrasta con la concepción romántica, que intenta rectificar. Pero también es radicalmente distinta a algunas de las posturas que florecieron como consecuencia del abandono de la propuesta hegeliana. Según Polo,

“el irracionalismo de ciertas corrientes vitalistas es el resultado de sacudirse la generalidad de la síntesis hegeliana y de no aceptar tampoco para la vida la lógica 
formal. Este vitalismo viene a ser una descomposición de Hegel" (Polo 2009, p. 140).

Como hemos visto, Hegel se propone una lucha enconada contra la irracionalidad de lo psíquico y el abandono a una afectividad irracional. Se puede decir que en esta empresa su rival es el empirismo y, sobre todo, Hume. Hegel tampoco hace preceder el sujeto a las ideas, sino que lo considera un resultado del proceso. Pero este proceso no es arbitrario: no puede ser acusado de psicologismo, porque es la Lógica misma. Ahora bien, recordémoslo bien, el precio que Hegel tiene que pagar es resignarse a aceptar la falsedad como un momento del proceso.

Los posthegelianos rechazarán a Hegel por distintas razones. Algunos rehabilitaron la positividad para hacerse cargo de aquello que Hegel no explica: el futuro. Hegel habla de una lógica generadora de contenidos, pero que sólo parece posible enunciar desde el estadio final. Ahora bien, si ya estamos en el final, la lógica no explica el futuro. Comte propone una visión positivista del saber, basada en el siguiente principio: "saber para prever, prever para poder". El conocimiento de las leyes que gobiernan los fenómenos nos permite predecir cómo se van a comportar en determinadas condiciones y nos ofrece la posibilidad de intervenir en ellos. Ésta es la gran promesa de la ciencia positiva y su baza frente al saber metafísico. Pero si la razón se identifica con la positividad, tampoco es posible hacerse cargo racionalmente de la espontaneidad. Esta incapacidad se refleja en la afectividad desbocada y desasosegada que domina la época, mientras que lo que Hegel intenta es apoderarse lógicamente de la espontaneidad: la pasión es una insatisfacción que orienta al yo hacia el infinito.

Una de las reacciones más vigorosas contra el positivismo como método racional único es la que se fragua en torno a unas ciencias que poco a poco van a alcanzar a lo largo del siglo XIX su madurez: las ciencias de la vida. "En torno a ellas", porque la aspiración de estas ciencias era precisamente alcanzar un estatuto positivo. El vitalismo, entendido en un sentido muy general, es una crítica de esta pretensión.

En Hegel la noción de vida es importante y es incompatible con la lógica formal. La vida es un movimiento de contenidos encerrado en el universal. Como afirma Polo, "la vida en Hegel no es positiva sino la supresión lograda de lo positivo" (L. Polo 2009, p. 139). Hegel nos habla de la vida al final de su Ciencia de la lógica, cuando trata de la Idea. La vida es la Idea inmediata, el concepto que es adecuado a su objetividad (cfr. G. W. F. Hegel [SWV, 249] 1982 , p. 483). Nos interesa ahora en particular la relación entre la vida y el espíritu: 
"En el espíritu, empero, la vida aparece por una parte como opuesta al espíritu mismo, por otra parte, como puesta juntamente con él, y esta unidad resulta a su vez generada por él. Es decir que aquí la vida tiene que ser entendida, en general, en su propio sentido, como vida natural, pues lo que se llama vida del espíritu como espíritu, es su peculiaridad, que se contrapone a la simple vida; así como se habla también de naturaleza del espíritu, a pesar de que el espíritu no es algo natural, y es más bien lo opuesto de la naturaleza" (G. W. F. Hegel [SW V, 246] 1982, p. 481).

Es difícil no recordar la tríada neoplatónica: alma, intelecto (o espíritu) y Uno. Hegel ofrece una interpretación distinta, pero no se separa demasiado de ella. Para Hegel la vida puede aplicarse en cierto sentido al espíritu, pero en otro, el espíritu es opuesto a lo meramente vital. Plotino, en cambio, con su visión gradual, no enfrenta en modo alguno el espíritu a la vida. Es más, por lo que hace a la noción, el espíritu es, en realidad, la vida perfecta (cfr. Plotino, Enéadas, I, 4, 3 et al.). Aunque Plotino no se atreve a aplicarla al Uno, a la primera hipóstasis. En esto se diferencia de la teología cristiana posterior, que, apoyándose en el carácter trino del Origen absoluto, no dudará en considerarlo también vida y no sólo origen de la vida. En esta estela parece situarse también Hegel, que, a pesar de las precisiones que hemos citado, no duda en aplicar la vida a la Idea absoluta:

"Sólo la Idea absoluta es ser, vida imperecedera, verdad que se conoce a sí misma, y es toda la verdad" (G. W. F. Hegel [SW V, 484] 1982, p. 725).

Bergson pone de manifiesto el carácter espacializante y analítico de la racionalidad positiva y su inadecuación para entender los fenómenos vitales. Junto a ella propone un método distinto, la intuición, que es capaz de captar el tiempo real en el que las diversas partes aparecen unidas. El impulso o élan vital vuelve a aparecer como algo ajeno a la razón, aunque no como algo totalmente ininteligible, dentro de un intento de rehabilitar el espíritu y la metafísica frente al monopolio de la razón científica. Por otra parte, en el mismo campo de la biología, Hans Driesch propone un principio distinto de las causas mecánicas, que son las que capta el método positivo de la biología de su tiempo. Es lo que él denomina "entelequia".

Ambos autores son importantes en la formación e inspiración de Max Scheler. Apoyados en la interpretación de Polo, nos vamos a detener en algunos aspectos de la propuesta de su última época, en la que recupera los motivos del idealismo dentro de su proyecto inconcluso de elaborar una antropología filosófica.

En El puesto del hombre en el cosmos Scheler define el espíritu desde la capacidad de ideación que encontramos en el ser humano, pero no como la manifestación máxima de la vida, sino como lo máximamente contrapuesto a 
ella. La vida estriba para Scheler en el impulso afectivo (Gefühlsdrang), que encontramos en todos los seres vivos desde los más elementales hasta los más complejos, y en todas la formas en que la vida se manifiesta: sea vegetativa o animal.

Scheler propone una escala de las formas de vida que sigue como criterio las formas de conocimiento. El conocimiento psíquico sería imposible sin el impulso afectivo. Éste es previo al conocer, puesto que es la raíz de la excitabilidad. Sólo captamos aquello que es relevante desde el impulso afectivo que da unidad al viviente. En el grado más alto del conocimiento, se encuentra la inteligencia práctica orgánicamente determinada (M. Scheler 2000, p. 59). Los animales que la alcanzan son capaces de comprender

"una situacion (conforme a su naturaleza contingente) en virtud de un sistema de referencia cuyos fundamentos están dados en parte por la experiencia y en parte son completados por anticipación de la representación" (M. Scheler 2000, p. 60).

No se trata sólo de una inteligencia asociativa, sino de algo más, que incluye la capacidad de establecer inferencias.

Sin embargo, el espíritu no sólo se distingue, sino que se opone a todas las formas vitales de conocimiento. La ideación enfrenta al hombre precisamente ante los objetos.

"El animal no tiene «objetos»: vive extáticamente en su medio, que por así decirlo lleva consigo allí donde va, como el caracol lleva incorporada su casa en forma de estructura, no es capaz de convertir este medio en objeto. Ese peculiar alejamiento, ese distanciamiento del «medio» que deviene «mundo» (o, más exactamente un símbolo del mundo) del que el hombre es capaz, no puede llevarlo a cabo el animal, incapaz de transformar en «objetos» los centros de resistencia delimitados afectiva e impulsivamente. Ser-objeto es, pues la categoría más formal de la vertiente lógica del espíritu" (M. Scheler 2000, p. 70; en la traducción que usamos hay un lapsus y se dice "estáticamente").

La nota fundamental del espíritu es, para Scheler, la capacidad de separar la esencia de la existencia (cfr. M. Scheler 2000, p. 82). A él corresponde la esfera de los valores y de las verdades puras. Pero el espíritu mismo es inobjetivable.

"Todo lo psíquico es susceptible de objetivación, no así el acto del espíritu, la intentio, la contemplación de los procesos psíquicos. En el ser de nuestra persona sólo podemos recogernos, concentrarnos en él, pero no objetivarlo" (M. Scheler 2000, p. 78).

La persona no se capta como las ideas, porque en modo alguno es objeto. Es un plexo o entramado de actos al que sólo se puede acceder activamente 
mediante la co-realización.

Es tarea de la persona realizar el mundo ideal del espíritu en el mundo material. La persona es el centro del espíritu y consiste en un plexo ordenado de actos esencialmente determinado que se realiza permanentemente a sí mismo (cfr. M. Scheler 2000, p. 78). Pero esto no quiere decir que el espíritu sea una realidad personal. En esto encontramos ecos de un problema que aparece ya en Hegel. El espíritu absoluto hegeliano no es persona porque no entabla relación con nada externo. En Scheler, sin embargo, el espíritu no es persona por sí mismo porque de suyo es impotente. Hasta tal punto lo es que no se basta para captar la realidad efectiva. El hombre se enfrenta a la realidad sólo como lo opuesto a su impulso vital. Sin esa confrontación esa categoría no tendría sentido. Pero el espíritu es ajeno de suyo a esa confrontación.

El espíritu de Scheler no es, por tanto, creador y no puede identificarse sin más con el fundamento del universo. A ese fundamento alude Scheler, pero sin dar otra determinación que sus diversas manifestaciones: el impulso vital y el espíritu. Queda así escindido en dos el primer principio. Se trata de una escisión que es el motor de su despliegue. En él, es el impulso vital el que pone la fuerza sin el cual el espíritu resulta ineficaz, y es el espíritu el que pone la dirección, aquello que la persona, como ser a caballo entre la vida y el espíritu, está llamada a realizar en la historia.

\section{II}

Nos encontramos en Hegel y Scheler no sólo con dos concepciones diversas del espíritu, sino también con dos formas de entender su relación con la espontaneidad y con la vida. La postura scheleriana recuerda en cierto modo al Schelling del Sistema del idealismo trascendental. Para éste, el primer principio es la autoconciencia, pero la autoconciencia puede entenderse como generadora de la realidad sólo en la medida en que sintetiza dos actividades contrapuestas: la actividad real y la actividad ideal. La primera es la actividad infinita que lleva a la conciencia a salir de sí, mientras que la segunda es la tendencia a intuirse en esa infinitud. Esta dualidad de la conciencia se reproduce en su visión del absoluto (cfr. F. W. J. Schelling 1988, 6, § 3). En esta obra, Schelling sitúa la suprema captación del espíritu en la actividad artística. De un modo semejante, parece que Scheler atribuye la experiencia y la realidad del absoluto al proceso de su realización, que corre a cargo del hombre.

Lo que aquí nos interesa señalar es que en Scheler el impulso ha sido de nuevo expulsado del espíritu. De este modo, lo psíquico, que en Hegel queda asumido por la lógica - que es al mismo tiempo motor y estructura del proceso-, se separa de ella y, como en el psicologismo que Hegel combate, vuelve a situarse a la espalda del espíritu. Scheler, no obstante, lucha contra el psicologismo y lo hace subrayando la independencia del espíritu respecto del 
impulso vital. Las ideas y los valores no están en modo alguno a su merced en cuanto contenidos y esencias, pero la única manera de que sean puestos en la realidad consiste en que la voluntad pura, como atributo del espíritu, extraiga del impulso vital la fuerza para que se realicen. La lógica hegeliana ha sido sustituida por una actividad del espíritu que es impotente por sí misma para realizarse. A diferencia de Hegel, lo espiritual queda aquí separado del mundo y sólo puede conectar con él mediante el esfuerzo de las personas, el único punto en que el espíritu es capaz de sublimar las fuerzas ciegas del universo y orientarlas para realizarse efectivamente en la historia

\section{REFERENCIAS}

BERGSON, H., 1970: Oeuvres, Paris: P. U. F.

HEGEL, G. W. F., 1999: Enciclopedia de las ciencias filosóficas, Madrid: Alianza Editorial.

1982: Ciencia de la lógica, Buenos Aires: Solar.

MURILLO, J. I., "El nacimiento de la antropología griega. Polo y la concepción de Platón sobre el hombre", Studia Poliana, 2005, N 7, 7-23.

POHLENZ, M., 1992: Die Stoa. Geschichte einer geistiger Bewegung, Göttingen: Vandenhoek \& Ruprecht.

POLO, L., 2009: Curso de psicología general, Pamplona: Eunsa.

REALE, G., 1989: Storia della filosofia antica, V, Milano: Vita e Pensiero.

SCHELER, M., 2000: El puesto del hombre en el cosmos, Barcelona: Alba Editorial.

SCHELLING, F. W. J., 1988: Sistema del idealismo trascendental, Barcelona: Anthropos. 
